\title{
Suppressing Proton Decay in the Minimal SO(10) Model
}

\author{
Bhaskar Dutta*, Yukihiro Mimura*, and R.N. Mohapatra ${ }^{\dagger}$ \\ * Department of Physics, University of Regina, Regina, Saskatchewan S4S 0A2, Canada \\ ${ }^{\dagger}$ Department of Physics, University of Maryland, College Park, MD 20742, USA
}

(Dated: August 10, 2018)

\begin{abstract}
We show that in a class of minimal supersymmetric $\mathrm{SO}(10)$ models which have been found to be quite successful in predicting neutrino mixings, all proton decay modes can be suppressed by a particular choice of Yukawa textures. This suppression works for contributions from both left and right operators for nucleon decay and for arbitrary $\tan \beta$. The required texture not only fits all lepton and quark masses as well as CKM parameters but it also predicts neutrino mixing parameter $U_{e 3}$ and Dirac CP phase $\sin \left|\delta_{M N S}\right|$ to be 0.07-0.09 and 0.3-0.7 respectively. We also discuss the relation between the GUT symmetry breaking parameters for the origin of these textures.
\end{abstract}

\section{INTRODUCTION}

Recent experiments in neutrino physics which have established that neutrino masses are in the sub-eV range have provided a new reason for taking supersymmetric grand unified theories (SUSY GUTs) seriously. This has to do with using the seesaw mechanism to understand the extreme smallness of neutrino masses compared to charged fermion masses [1]. The seesaw mechanism consists of extending the standard model by introducing three right-handed neutrinos with large Majorana masses $M_{R}$. Simple arguments based on the atmospheric oscillation data then tell us that at least one $M_{R}$ is close to the conventional GUT scale $M_{U} \sim 10^{16} \mathrm{GeV}$, perhaps suggesting that the seesaw scale, $M_{R}$ and GUT scale are one and the same.

The minimal GUT that unifies the right-handed neutrinos with the other fermions of the standard model and leads to the seesaw mechanism is the $\mathrm{SO}(10)$ model where all quarks and leptons are unified into one $\mathbf{1 6}$ dimensional spinor multiplet. This raises the possibility that the variety of masses and mixings of quarks and leptons can be understood in terms of a smaller number of parameters than in the standard model with three right-handed neutrinos. In fact, various recent works in the minimal $\mathrm{SO}(10)$ models with a single $\mathbf{1 0}$ and a single $\overline{\mathbf{1 2 6}}$ Higgs multiplets [2, 3, 4, 5, 6] have substantiated this point of view. Unlike the generic seesaw models based on the extension of the standard model as well as right-handed neutrino extended $\mathrm{SU}(5)$ model, the above $\mathrm{SO}(10)$ models have fewer parameters and lead to predictions for neutrino mixings and phases in their minimal version without any extra symmetry assumptions. Their predictions for solar and atmospheric mixing angles are in agreement with present observations [5, 6] and that for $U_{e 3}$ is not far below the present upper limits, making the model testable in planned experiments [7]. Measurement of $U_{e 3}$ will therefore provide a crucial test of these models.

It has further been shown that the simplest way to accommodate CKM CP violation in these models is to include an additional Higgs field belonging to the $\mathbf{1 2 0}$ dimensional representation [8]. The model with $\mathbf{1 2 0}$ still remains predictive in the neutrino sector despite a small increase in the number of parameters 8,9$]$ and also leads to a solution to the SUSY CP problem.

Since the quarks and leptons are unified in a GUT, it predicts that proton is no longer stable and therefore proton decay becomes another test of any GUT such as $\mathrm{SO}(10)$. The present experimental bounds on the proton lifetime are in fact known to severely constrain some GUT models [10, 11] and one must therefore make sure that the above class of $\mathrm{SO}(10)$ models are in agreement with present experimental results.

In most generic SUSY GUTs, the dimension five operators induced by colored Higgsino [12] provide the dominant contribution to the proton decay amplitude rather than the dimension six operators induced by gauge bosons. Since the dimension five operators arise from diagrams involving Yukawa couplings, predictions for proton lifetime get related to fermion mass textures. For example, in simple GUT theories such as minimal SU(5), where by minimal we mean that only the most general renormalizable terms are included in the superpotential, the up- and down-type quark mass matrices are proportional to the Yukawa matrices, $Y_{u}$ and $Y_{d}$ respectively. In this case the proton decay rate, $\tau_{p}^{-1}$ is directly proportional to $\left|Y_{u, i j} Y_{d, k l}\right|^{2}$ implying that $\tau_{p}$ cannot be arbitrarily adjusted. That is why the minimal SU(5) theory is now ruled out by proton decay results 11].

One might argue that the minimal SU(5) model is anyway not realistic since it predicts wrong relations between fermion masses e.g. $m_{s}=m_{\mu}$ and $m_{d}=m_{e}$. This fermion mass problem is however easily remedied in the class of minimal $\mathrm{SO}(10)$ models [2] with a single $\mathbf{1 0}$ and $\overline{\mathbf{1 2 6}}$ field mentioned above. In view of the fact that it is also quite predictive in the neutrino sector 2, 4, 5, 6], it is tempting to consider this as the minimal GUT comparable to what SU(5) GUT was in the 1980's. It is then important to look for its predictions for proton decay. To be sure, this model like minimal SU(5) (and the standard model) does not explain the origin of the Yukawa couplings; however if this model is confirmed by experiments, understanding the Yukawa sector will be the next order of business. 
Unlike the $\mathrm{SU}(5)$ model, the $\Delta B=1$ interactions in generic $\mathrm{SO}(10)$ models 13 involve more GUT scale symmetry breaking parameters than just the color triplet Higgsino mass; therefore the situation for proton decay here is less restrictive. In particular, in the minimal $\mathrm{SO}(10)$ models of the type discussed in Ref. [5] , there are four free parameters 14 whereas there are about 16 decay modes which have lower bounds on their partial life times. It was shown through a numerical analysis 14, 15. (without including $R R R R$ operators) that there exists a very small region in these parameter spaces for $L L L L$ operators, where all the present experimental constraints are satisfied for lower $\tan \beta$.

In this paper, we study the proton decay constraints on $\mathrm{SO}(10)$ models with $\mathbf{1 0}+\overline{\mathbf{1 2 6}}+\mathbf{1 2 0}$ Higgs fields. We show that present proton decay constraints combined with fermion masses and mixings imply a specific relation among $\mathrm{SO}(10)$ breaking vacuum expectation values (VEVs) and a very specific form for the Yukawa textures. Roughly, they imply that the proton decay operators are proportional to the product of two up-type quark Yukawa couplings i.e. $Y_{u, i j} Y_{u, k l}$ instead of $Y_{d} Y_{u}$ as in $\mathrm{SU}(5)$. As a consequence, proton decay is not only suppressed for the left-handed quark contributions but also for right-handed ones and furthermore, the suppression works also for large $\tan \beta$ values as well which makes this model easily distinguishable from other simple GUT models. In addition, it leads to definite predictions for the neutrino mixing parameters $U_{e 3}$ and the Dirac phase $\delta_{M N S}$. We also show the essential role played by the $\mathbf{1 2 0}$ in the suppression of proton decay when all operators are taken into account. These are the new results of this paper, which have important implications for the viability of the minimal $\mathrm{SO}(10)$ model for neutrino masses with CKM CP violation.

\section{FERMION MASS AND PROTON DECAY IN MINIMAL SO(10)}

We start by introducing the Yukawa interactions and the contents of Higgs fields in the $\mathrm{SO}(10)$ model. The Yukawa superpotential involves the couplings of 16dimensional matter spinors $\psi_{i}(i$ denotes a generation index) with $10(H), \overline{\mathbf{1 2 6}}(\bar{\Delta})$, and $\mathbf{1 2 0}(D)$ dimensional Higgs fields:

$$
W_{Y}=\frac{1}{2} h_{i j} \psi_{i} \psi_{j} H+\frac{1}{2} f_{i j} \psi_{i} \psi_{j} \bar{\Delta}+\frac{1}{2} h_{i j}^{\prime} \psi_{i} \psi_{j} D .
$$

The Yukawa couplings, $h$ and $f$ are symmetric matrices, whereas $h^{\prime}$ is an anti-symmetric matrix due to $\mathrm{SO}(10)$ symmetry. One 126 Higgs multiplet $\Delta$ is also introduced as a vector-like pair of $\bar{\Delta}$ whose VEV reduces the rank of SO(10) group. This helps to keep supersymmetry unbroken down to the weak scale. In order to break $\mathrm{SO}(10)$ symmetry down to the standard model, we employ one
210 Higgs field $(\Phi)$ 16] which also contains a pair of Higgs doublets $\left(\Phi_{u}, \Phi_{d}\right)$. Altogether, we have six pairs of Higgs doublets: $\varphi_{d}=\left(H_{d}^{10}, D_{d}^{1}, D_{d}^{2}, \bar{\Delta}_{d}, \Delta_{d}, \Phi_{d}\right), \varphi_{u}=$ $\left(H_{u}^{10}, D_{u}^{1}, D_{u}^{2}, \Delta_{u}, \bar{\Delta}_{u}, \Phi_{u}\right)$, where superscripts 1,2 of $D_{u, d}$ stand for $\mathrm{SU}(4)$ singlet and adjoint pieces under the $G_{422}=\mathrm{SU}(4) \times \mathrm{SU}(2) \times \mathrm{SU}(2)$ decomposition. The mass term of the Higgs doublets is given as $\left(\varphi_{d}\right)_{a}\left(M_{D}\right)_{a b}\left(\varphi_{u}\right)_{b}$, and the expression of the matrix $M_{D}$ is given in Ref. [17]. The mass matrix of the Higgs doublets is diagonalized by unitary matrices $U$ and $V: U M_{D} V^{\mathrm{T}}=M_{D}^{\text {diag }}$. We assume that $\left(M_{D}^{\text {diag }}\right)_{11}=\mu$, where $\mu$ is a Higgsino mass in the MSSM and the mass scale is much smaller than the GUT scale. Since we concentrate on the structure of Yukawa couplings, we do not need to specify the dynamical reason of the mass hierarchy in this letter, but we just require a fine-tuning such as in the case of minimal SU(5) model[10]. The MSSM Higgs doublets are given as linear combinations: $H_{d}=U_{1 a}^{*}\left(\varphi_{d}\right)_{a}, H_{u}=V_{1 a}^{*}\left(\varphi_{u}\right)_{a}$.

We use "Y diagonal basis" (or SU(5) basis) to describe the standard model decomposition of the $\mathrm{SO}(10)$ representation [17, 18]. The expression of the Yukawa interaction under the $G_{422}$ decomposition can be derived from Ref.[19]. The decomposed Yukawa interactions which give fermion masses are written as

$$
\begin{aligned}
& W_{Y}^{\text {doub. }}=h H_{d}^{10}\left(q d^{c}+\ell e^{c}\right)+h H_{u}^{10}\left(q u^{c}+\ell \nu^{c}\right) \\
& +\frac{1}{\sqrt{3}} f \bar{\Delta}_{d}\left(q d^{c}-3 \ell e^{c}\right)+\frac{1}{\sqrt{3}} f \bar{\Delta}_{u}\left(q u^{c}-3 \ell \nu^{c}\right) \\
& +h^{\prime} D_{d}^{1}\left(q d^{c}+\ell e^{c}\right)+h^{\prime} D_{u}^{1}\left(q u^{c}+\ell \nu^{c}\right) \\
& +\frac{1}{\sqrt{3}} h^{\prime} D_{d}^{2}\left(q d^{c}-3 \ell e^{c}\right)-\frac{1}{\sqrt{3}} h^{\prime} D_{u}^{2}\left(q u^{c}-3 \ell \nu^{c}\right),
\end{aligned}
$$

where $q, u^{c}, d^{c}, \ell, e^{c}, \nu^{c}$ are the quark and lepton fields for the standard model, which are all unified into one spinor representation of $\mathrm{SO}(10)$. We obtain the Yukawa coupling matrices for fermions as

$$
\begin{aligned}
Y_{u} & =\bar{h}+r_{2} \bar{f}+r_{3} \bar{h}^{\prime}, \\
Y_{d} & =r_{1}\left(\bar{h}+\bar{f}+\bar{h}^{\prime}\right), \\
Y_{e} & =r_{1}\left(\bar{h}-3 \bar{f}+c_{e} \bar{h}^{\prime}\right), \\
Y_{\nu} & =\bar{h}-3 r_{2} \bar{f}+c_{\nu} \bar{h}^{\prime},
\end{aligned}
$$

where the subscripts $u, d, e, \nu$ denotes for up-type quark, down-type quark, charged-lepton, and Dirac neutrino Yukawa couplings, respectively and

$$
\begin{aligned}
\bar{h} & =V_{11} h, r_{1}=U_{11} / V_{11}, r_{2}=r_{1} V_{15} / U_{14}, \\
r_{3} & =r_{1}\left(V_{12}-V_{13} / \sqrt{3}\right) /\left(U_{12}+U_{13} / \sqrt{3}\right), \\
\bar{f} & =U_{14} /\left(\sqrt{3} r_{1}\right) f, \bar{h}^{\prime}=\left(U_{12}+U_{13} / \sqrt{3}\right) / r_{1} h^{\prime}, \\
c_{e} & =\left(U_{12}-\sqrt{3} U_{13}\right) /\left(U_{12}+U_{13} / \sqrt{3}\right), \\
c_{\nu} & =r_{1}\left(V_{12}+\sqrt{3} V_{13}\right) /\left(U_{12}+U_{13} / \sqrt{3}\right) .
\end{aligned}
$$

The Majorana mass matrices for both left- and righthanded neutrinos are proportional to the coupling $f$. In this letter, we will be using type II seesaw 20]. 
Next we consider dimension five operators induced by Higgs triplets. The dimension five operators $(L L L L$ and $R R R R$ operators),

$$
-W_{5}=\frac{1}{2} C_{L}^{i j k l} q_{k} q_{l} q_{i} \ell_{j}+C_{R}^{i j k l} e_{k}^{c} u_{l}^{c} u_{i}^{c} d_{j}^{c},
$$

are obtained by integrating out the triplet Higgs fields, $\varphi_{\bar{T}}=\left(H_{\bar{T}}, D_{\bar{T}}, D_{\bar{T}}^{\prime}, \bar{\Delta}_{\bar{T}}, \Delta_{\bar{T}}, \Delta_{\bar{T}}^{\prime}, \Phi_{\bar{T}}\right)$ and $\varphi_{T}=$ $\left(H_{T}, D_{T}, D_{T}^{\prime}, \Delta_{T}, \bar{\Delta}_{T}, \bar{\Delta}_{T}^{\prime}, \Phi_{T}\right)$. The quantum numbers under $\mathrm{SU}(3) \times \mathrm{SU}(2) \times \mathrm{U}(1)_{Y}$ of the field $\varphi_{T}$ is $(\mathbf{3}, \mathbf{1},-1 / 3)$. In the expression of $\varphi_{T}$, the fields with " are decuplet, and the others are sextet under $\mathrm{SU}(4)$ decomposition. The $R R R R$ operator, $C_{R}$, is also generated by other colored triplet, $\varphi_{\bar{C}}=\left(D_{\bar{C}}, \Delta_{\bar{C}}\right)$ and $\varphi_{C}=\left(D_{C}, \bar{\Delta}_{C}\right)$, where the quantum number of $\varphi_{C}$ is $(\mathbf{3}, \mathbf{1},-4 / 3)$. The mass term of the Higgs triplets are given as $\left(\varphi_{\bar{T}}\right)_{a}\left(M_{T}\right)_{a b}\left(\varphi_{T}\right)_{b}+\left(\varphi_{\bar{C}}\right)_{a}\left(M_{C}\right)_{a b}\left(\varphi_{C}\right)_{b}$. The mass matrices, $M_{T}$ and $M_{C}$, are $7 \times 7$ and $2 \times 2$ matrices respectively, and their explicit forms are given in the literature [17]. The Yukawa couplings which cause proton decay are written as

$$
\begin{aligned}
& W_{Y}^{\text {trip. }}=h H_{\bar{T}}\left(q \ell+u^{c} d^{c}\right)+h H_{T}\left(\frac{1}{2} q q+e^{c} u^{c}\right) \\
+ & f \bar{\Delta}_{\bar{T}}\left(q \ell-u^{c} d^{c}\right)+f \bar{\Delta}_{T}\left(\frac{1}{2} q q-e^{c} u^{c}\right)+\sqrt{2} f \bar{\Delta}_{T}^{\prime} e^{c} u^{c} \\
+ & \sqrt{2} h^{\prime} D_{\bar{T}} u^{c} d^{c}+\sqrt{2} h^{\prime} D_{\bar{T}}^{\prime} q \ell \\
& -\sqrt{2} h^{\prime} D_{T} e^{c} u^{c}+\sqrt{2} h^{\prime} D_{T}^{\prime} e^{c} u^{c} \\
+ & 2 f \bar{\Delta}_{C} d^{c} e^{c}+2 h^{\prime} D_{\bar{C}} u^{c} u^{c}+2 h^{\prime} D_{C} d^{c} e^{c} .
\end{aligned}
$$

The dimension five operators are written by the Yukawa couplings $h, f$ and $h^{\prime}$ as follow:

$$
\begin{aligned}
& C_{L}^{i j k l}=c h_{i j} h_{k l}+x_{1} f_{i j} f_{k l}+x_{2} h_{i j} f_{k l}+x_{3} f_{i j} h_{k l} \\
& +x_{4} h_{i j}^{\prime} h_{k l}+x_{5} h_{i j}^{\prime} f_{k l}, \\
& C_{R}^{i j k l}=c h_{i j} h_{k l}+y_{1} f_{i j} f_{k l}+y_{2} h_{i j} f_{k l}+y_{3} f_{i j} h_{k l} \\
& +y_{4} h_{i j}^{\prime} h_{k l}+y_{5} h_{i j}^{\prime} f_{k l}+y_{6} h_{i j} h_{k l}^{\prime}+y_{7} f_{i j} h_{k l}^{\prime}+y_{8} h_{i j}^{\prime} h_{k l}^{\prime} \\
& +y_{9} h_{i l}^{\prime} f_{j k}+y_{10} h_{i l}^{\prime} h_{j k}^{\prime} .
\end{aligned}
$$

The common coefficient $c$ is given as $c=\left(M_{T}^{-1}\right)_{11}$. We obtain the other coefficients as the following:

$$
\begin{aligned}
& \left(x_{1}, x_{2}, x_{3}, x_{4}, x_{5}\right) \\
= & \left(M_{54}^{-1}, M_{51}^{-1}, M_{14}^{-1}, \sqrt{2} M_{13}^{-1}, \sqrt{2} M_{53}^{-1}\right), \\
& \left(y_{1}, y_{2}, y_{3}, y_{4}, y_{5}, y_{6}, y_{7}, y_{8}\right) \\
= & \left(M_{54}^{-1}-\sqrt{2} M_{64}^{-1},-M_{51}^{-1}+\sqrt{2} M_{61}^{-1},-M_{14}^{-1},\right. \\
& \sqrt{2} M_{12}^{-1},-\sqrt{2} M_{52}^{-1}+2 M_{62}^{-1}, \sqrt{2}\left(-M_{21}^{-1}+M_{31}^{-1}\right), \\
& \left.\sqrt{2}\left(M_{24}^{-1}-M_{34}^{-1}\right), 2\left(-M_{22}^{-1}+M_{32}^{-1}\right)\right),
\end{aligned}
$$

where subscript $T$ of the matrix $M_{T}$ is omitted. It is important to note that $y_{3}=-x_{3}$, namely their signatures are opposite between $L L L L$ and $R R R R$ operator. This is derived from the fact that $H_{T}$ and $\bar{\Delta}_{T}$ have opposite
D-parity. The coefficients $y_{9}, y_{10}$ are generated by $\varphi_{C}$, $\varphi_{\bar{C}}$, and $y_{9}=4\left(M_{C}^{-1}\right)_{21}, y_{10}=4\left(M_{C}^{-1}\right)_{11}$.

The Eqs. (14 17) are explicit forms of the dimension five operators, but we present here more convenient forms to describe proton decay. The Higgs triplet mass matrix $M_{T}$ is diagonalized by two unitary matrices, $X$ and $Y$, as $X M_{T} Y^{\mathrm{T}}=\operatorname{diag}\left(M_{1}, M_{2}, \cdots, M_{7}\right)$. Then we obtain the useful formula for dimension five operators

$$
\begin{aligned}
& C_{L}^{i j k l}=\sum_{a} \frac{1}{M_{a}}\left(X_{a 1} h+X_{a 4} f+\sqrt{2} X_{a 3} h^{\prime}\right)_{i j} \\
& \times\left(Y_{a 1} h+Y_{a 5} f\right)_{k l}, \\
& C_{R}^{i j k l}=\sum_{a} \frac{1}{M_{a}}\left(X_{a 1} h-X_{a 4} f+\sqrt{2} X_{a 2} h^{\prime}\right)_{i j} \\
& \times\left(Y_{a 1} h-\left(Y_{a 5}-\sqrt{2} Y_{a 6}\right) f+\sqrt{2}\left(Y_{a 3}-Y_{a 2}\right) h^{\prime}\right)_{k l} \\
& +\left(y_{9}, y_{10} \text { terms }\right) \text {. }
\end{aligned}
$$

One can make consistency checks to verify the formula by considering specific vacua of the theory. For example, in the SU(5) limit, when only one of the colored triplets is much lighter than the others, i.e. $M_{1} \ll M_{a}(a \neq 1)$, we can obtain the following relations for the diagonalizing matrices from the explicit form of the Higgs mass matrices with the $\mathrm{SU}(5)$ vacua in the Ref. 17, 18]:

$$
\begin{aligned}
U_{11}=X_{11}, V_{11} & =Y_{11}, U_{14}=X_{14}=0 \\
V_{15}: Y_{15}: Y_{16} & =\sqrt{3}: 1: \sqrt{2} \\
U_{12}: U_{13}: X_{12}: X_{13} & =V_{12}: V_{13}: Y_{12}: Y_{13} \\
& =1: \sqrt{3}: \sqrt{2}: \sqrt{2}
\end{aligned}
$$

As a result, $r_{2} \rightarrow \infty$ with $\bar{f} \rightarrow 0$,

$$
r_{3}=0, c_{e}=-1
$$

for the Yukawa matrices in Eqs. (36) and thus, as expected, we get the $\mathrm{SU}(5)$ relations, $Y_{u}=Y_{u}^{\mathrm{T}}, Y_{d}=Y_{e}^{\mathrm{T}}$, and the dimension five proton decay operators can be written in terms of the Yukawa couplings as

$$
C_{L}^{i j k l} \simeq C_{R}^{i j k l} \simeq\left(Y_{d}\right)_{i j}\left(Y_{u}\right)_{k l} / M_{1} .
$$

One can also obtain the flipped-SU(5) limit 21 in a similar manner and find the relations, $Y_{u}=Y_{\nu}^{\mathrm{T}}, Y_{d}=$ $Y_{d}^{\mathrm{T}}$, and

$$
C_{L}^{i j k l} \simeq\left(Y_{u}\right)_{j i}\left(Y_{d}\right)_{k l} / M_{1}, C_{R}^{i j k l} \simeq\left(Y_{u}\right)_{i j}\left(Y_{e}\right)_{l k} / M_{1} \cdot(26)
$$

One can also consider other typical vacua such as the one corresponding to $G_{422}$. On this vacuum, we have $U_{13}=V_{13}=U_{14}=V_{15}=0$ and all four fermion Yukawa couplings are unified as $Y_{u, d, e, \nu}=\bar{h}+\bar{h}^{\prime}$, which is not viable for phenomenology. In any case, since the doublet and triplet Higgs fields are not unified in one multiplet on this vacuum, proton decay operator cannot be written in terms of fermion Yukawa couplings contrary to the $\mathrm{SU}(5)$ and flipped-SU(5) limit. The operators $C_{L, R}^{i j k l}$ in the $G_{422}$ 
limit are symmetric under the interchange of indices $i j$ and $k l$ in addition to the symmetry under the exchange of individual indices $i$ and $j$. One can write down the dimension five operators as

$$
C_{L} \propto(h+z f)(h+z f), C_{R} \propto(h-z f)(h-z f),
$$

with $z=X_{14} / X_{11}$. It is important to stress that there are relatively opposite signatures for the $f$ contributions between $C_{L}$ and $C_{R}$. This is due to D-parity, which we have already mentioned.

\section{SUPPRESSION OF PROTON DECAY}

Let us now investigate the conditions required to suppress the proton decay rate in the minimal $\mathrm{SO}(10)$ model described above. We first note that the four-fermion proton decay operators are produced by gaugino and Higgsino dressing of the dimension five operators. The fourfermion operators of $L L L L$ type get dominant contribution from wino exchange and therefore retain the same flavor structure as that of the original dimension five supersymmetric operator (we have assumed universality of scalar masses for suppressing FCNC processes). However, as far as the $R R R R$ operators are concerned, they receive contributions only from Higgsino exchange which can involve the top quark and $\tau$ lepton Yukawa couplings. For instance in the case of $\mathrm{SU}(5)$ model, the contribution of the $R R R R$ operator to the decay mode $p \rightarrow K \bar{\nu}_{\tau}$ gets so enhanced because of this that it exceeds the current experimental bound for any $\tan \beta$ as long as stop mass is less than around $1 \mathrm{TeV}[1]$.

Since the original Yukawa couplings $h, f$, and $h^{\prime}$ are functions of fermion Yukawa couplings $Y_{u}, Y_{d}$ and $Y_{e}$ via Eqs.(35), their textures are roughly determined from the experimental inputs of quark and lepton masses and mixings. For instance, to fit the strange quark and muon masses, bottom-tau unification, relations among CKM mixings and also large mixings of neutrinos, the coupling $f$ is almost determined to have the form $\bar{f} \sim$ $\left(\begin{array}{ccc}\lambda^{2} & \lambda & \lambda \\ \lambda & 1 & 1 \\ \lambda & 1 & 1\end{array}\right) m_{s} / m_{b}$, where $\lambda \sim 0.2$. A naive implication of this is that since up-type quark masses are more hierarchical than down-type ones (i.e. $m_{u} / m_{t} \ll$ $\left.m_{d} / m_{b}, m_{c} / m_{t} \ll m_{s} / m_{b}\right)$, the expression for the uptype Yukawa matrix, $Y_{u}$, in Eq. (3) requires the following two typical choices: (a) there is cancellation among $h, f$, and $h^{\prime}$, or (b) $h$ itself has a hierarchical form similary to the up-type quark masses. The first choice (a) is the case where $[1,2]$ block of $h_{i j}$ is not far smaller than $f_{i j}$, but $r_{2,3}$ are chosen to be certain values to make $m_{u}, m_{c}$ are hierarchically small. The second choice (b) corresponds to the case where $r_{2,3} \sim 0$. The second choice appears to be required to suppress the proton decay. Let us discuss the reason.
In order to suppress the decay rate, we need small couplings for first and second generations in the expressions in Eqs. 14 15. Clearly this would also require a cancellation among $h, f$ and $h^{\prime}$ if we take the first choice (a). Since in general the coefficients $r_{2}, r_{3}$ in up-type Yukawa matrix and $x_{i}$ and $y_{i}$ in proton decay operators are unrelated, one must find a situation where both cancellations can be achieved in a satisfactory manner so as to be consistent with all data. However, if we take the choice (a), the cancellation cannot happen naturally due to the following reasons.

First, let us discuss the $\overline{\mathbf{1 2 6}}$ Higgs contribution. Since there is an opposite signature for one of the coefficients in the $L L L L$ and $R R R R$ operators, $y_{3}=-x_{3}$, the cancellation required to obtain small Yukawa coupling for $Y_{u}$ by tuning $r_{2} \bar{f}$ cannot simultaneously suppress both $L L L L$ and $R R R R$ operators. Thus, in general, it is hard to suppress proton decay rate by tuning $\overline{\mathbf{1 2 6}}$ colored Higgs mixing $X_{14}$. Next let us see the contribution from $\mathbf{1 2 0}$ Higgs field. The coefficient $C_{L}^{i j k l}$ is symmetric in the indices $k l$ due to $\mathrm{SU}(3) \times \mathrm{SU}(2)$ contraction. Therefore $h^{\prime}$ contribution is absent for the $q_{k} q_{l}$ part in $L L L L$ operator, whereas it is of course present in the fermion masses. Thus, if the cancellation in $Y_{u}$ happens by tuning $r_{3} h^{\prime}$, such cancellation will not help in suppressing the $L L L L$ operator.

The above discussions lead to the fact that the proton decay rate cannot be suppressed in natural way if we take the choice (a). As we will see numerical studies later, we require a fine-tuning to the level of $0.01 \%$ to suppress all the proton and neutron decay modes when we consider general parameter fitting in the choice (a). The choice (b) $\left(r_{2,3} \simeq 0\right)$ is necessary to achieve natural suppression of proton decay as a result.

We now show how the proton decay rate in the choice (b) is suppressed rather than the minimal $\mathrm{SU}(5)$. If $r_{2,3} \simeq 0$, the $L L L L$ dimension five operator can be a form as $C_{L}^{i j k l} \propto\left(Y_{u}+\gamma h^{\prime}\right)_{i j}\left(Y_{u}\right)_{k l}$ and in the $R R R R$ operator $C_{R}^{i j k l}, k l$ part is also related to $Y_{u}$. This will correspond to the case where $X_{14}, Y_{15} \sim 0$. We will give an example later where this can happen but first we note that for the case just noted, the $R R R R$ contribution to $p \rightarrow K \bar{\nu}_{\tau}$ mode is suppressed compared to the usual SU(5) models for the entire range of $\tan \beta$ up to 50. Here, the dominant contribution is proportional to $\lambda_{u}$ giving a suppression factor $\lambda_{u} / \lambda_{d} \sim 1 / 100$ for $\tan \beta \sim 50$ compared to minimal $\mathrm{SU}(5)$ case. Similarly, since the $k l$ part of $C_{L}$ are also related to the $Y_{u}$ instead of $Y_{d}$, the $L L L L$ contribution to the $p \rightarrow K \bar{\nu}$ is also suppressed even for $\tan \beta \sim 50$, compared to the SU(5) model (since $\left.\lambda_{c} / \lambda_{s} \sim 1 / 5\right)$. However as it turns out, these suppressions are not enough. We need to specify the Yukawa texture for the purpose as we see below.

Before describing the specific choice of Yukawa textures, let us show the numerical values for the pro- 
TABLE I: Typical proton decay amplitude for different values of $r_{2}$ and $r_{3}$. To satisfy current experimental bound naturally, $\left|\tilde{A}_{h h}\right| \lesssim 10^{-8}$ is needed.

\begin{tabular}{|c|c|}
\hline \hline$r_{2}, r_{3}$ & $\tilde{A}_{h h}$ \\
\hline$r_{2}=r_{3}=0$ & $(4.2+0.083 i) \times 10^{-7}$ \\
$r_{2}=0, r_{3}=1$ & $(1.4-0.17 i) \times 10^{-4}$ \\
$r_{2}=0.5, r_{3}=0$ & $(7.3+2.6 i) \times 10^{-5}$ \\
\hline \hline
\end{tabular}

ton decay amplitude to confirm that $r_{2,3} \simeq 0$ is necessary to satisfy the current experimental bounds naturally. Rewriting the proton decay amplitude as $A=$ $\alpha_{2} \beta_{p} /\left(4 \pi M_{T} m_{S U S Y}\right) \tilde{A}$, we define

$$
\tilde{A}=c \tilde{A}_{h h}+x_{1} \tilde{A}_{f f}+x_{2} \tilde{A}_{h f}+x_{3} \tilde{A}_{f h}+x_{4} \tilde{A}_{h^{\prime} h}+\cdots
$$

The coefficients $c$ and $x_{i}$ are given in Eq.(14). The $R R R R$ contribution can be written in the same way. To satisfy the current proton and neutron decay bounds, we need $\left|\tilde{A}_{p \rightarrow K \bar{\nu}}\right| \lesssim 10^{-8},\left|\tilde{A}_{n \rightarrow \pi \bar{\nu}}\right| \lesssim 2 \cdot 10^{-8}$ and $\left|\tilde{A}_{n \rightarrow K \bar{\nu}}\right| \lesssim 5 \cdot 10^{-8}$ if the colored Higgsino mass is $2 \cdot 10^{16}$ $\mathrm{GeV}$, and squark and wino masses are around $1 \mathrm{TeV}$ and $250 \mathrm{GeV}$, respectively. In order to satisfy those bounds naturally, we need $\tilde{A}_{h h} \lesssim 5 \cdot 10^{-8}$. If $\tilde{A}_{h h} \gtrsim 10^{-7}$, we need to tune $x_{i}$ and $y_{i}$ for every decay mode to cancel $\tilde{A}_{h h}$, which is unnatural. (Further, assuming $c \rightarrow 0$ cannot make successful suppression of the decay amplitude since in that assumption we also need to suppress $\tilde{A}_{f f}$ which can only be suppressed for $\tan \beta \lesssim 3$ ). The $\tilde{A}_{h h}$ depends on the magnitudes of the elements from the $[1,2]$ block of $\bar{h}$ which is determined from the fit to the up-type Yukawa as a function of $r_{2}$ and $r_{3}$. In Table show the $\tilde{A}_{h h}$ component in the proton decay amplitude for different values of $r_{3}$ and $r_{2}$. The $\tilde{A}_{h h}$ is calculated in the basis where $Y_{u}=\operatorname{diag}\left(\lambda_{u}, \lambda_{c}, \lambda_{t}\right)$. As we can see from the table, that $\tilde{A}_{h h}$ can be very large (for large $r_{2,3}$ ) and this requires very high level of fine-tuning for all the decay modes (including charged leptonic decay) to suppress nucleon decay as we noted. The smallness of $r_{2}$ and $r_{3}$ is required to suppress $\tilde{A}_{h h}$. However, even in the case $r_{2}=r_{3}=0$, the $\tilde{A}_{h h}$ amplitude is not enough suppressed $\left(\tilde{A}_{h h} \sim 10^{-7}\right)$ and we still need fine-tuning among the coefficients to satisfy the data. This is mainly because $\lambda_{u, c}$ are not enough small to satisfy $\tilde{A}_{h h} \lesssim 10^{-8}$.

We now present the specific choice of Yukawa texture to suppress the proton decay rate further. The texture is given as $\bar{h} \simeq \operatorname{diag}(0,0, O(1))$, so that up and charm quark masses arise from contributions of $f$ and $h^{\prime}$ :

$$
\bar{f} \simeq\left(\begin{array}{ccc}
\sim 0 & \sim 0 & \lambda^{3} \\
\sim 0 & \lambda^{2} & \lambda^{2} \\
\lambda^{3} & \lambda^{2} & \lambda^{2}
\end{array}\right), \bar{h}^{\prime} \simeq i\left(\begin{array}{ccc}
0 & \lambda^{3} & \lambda^{3} \\
-\lambda^{3} & 0 & \lambda^{2} \\
-\lambda^{3} & -\lambda^{2} & 0
\end{array}\right)
$$

where $\lambda \sim 0.2$. In this texture, $r_{3}=0$, and $r_{2}$ is fixed as $r_{2} m_{s} / m_{b} \simeq \lambda_{c}\left(r_{2} \simeq 0.1\right)$ to generate the correct charm mass. We have $f_{11,12} \sim 0$ (nonzero value of $r_{2}$ is therefore allowed) to ensure small values of $h_{11,12}$, and $h_{12}^{\prime}$ generates the down-quark mass and Cabibbo angle $\theta_{c}$ with $m_{d} / m_{s} \simeq \sin ^{2} \theta_{c}$. The up-quark Yukawa coupling is generated as $\lambda_{u} \sim\left(r_{2} \lambda^{3}\right)^{2}$, and we also have a relation $m_{d} m_{s} m_{b} \simeq c_{e}^{2} m_{e} m_{\mu} m_{\tau}$, where $c_{e}^{2} \simeq 1$ in the preferred vacuum for the 120 Higgs coupling, Eq.24. In the basis where $Y_{u}$ is diagonal, $\widetilde{A}_{h h}$ in this texture is not completely zero but can become much smaller than $10^{-8}$.

We comment that we also need to examine the contribution of the other components e.g. $\tilde{A}_{f f, h f, f h, h^{\prime} f, h^{\prime} h, \cdots}$ after we suppress the $\tilde{A}_{h h}$. However, their contributions are associated with the colored Higgs mixing angles, which are inputs and can be suppressed by our choice of the vacuum expectation values and the Higgs couplings. According to our numerical studies, some of the mixing angles must be about a few percent in the case of $\tan \beta \sim 50$ to suppress the decay. However, the mixing angles can become larger as $\tan \beta$ becomes smaller. Details will be given in a future paper 22].

Without the particular choice of texture, as mentioned above, the proton decay can not be suppressed naturally in these models unless the $\tan \beta$ is very small.

The proton life time for $p \rightarrow K \bar{\nu}$ for this choice of texture can be larger than the current experimental bound, $\tau_{p} \gtrsim 2 \cdot 10^{33}$ years for any $\tan \beta$ (using the lightest colored Higgsino mass to be $2 \cdot 10^{16} \mathrm{GeV}$ and squark mass scale around $1 \mathrm{TeV}$ ). All other nucleon decay modes are suppressed as well. In our calculation, we use long- and short-distance renormalization factor, $A_{L}=1.43$ and typically $A_{S}=1.8$ similar to Ref. 23].

Given the above texture for Yukawa couplings, we find that $U_{e 3}$ is restricted to a range. In figure 11 we plot $U_{e 3}$ as a function of $r_{2}$ with $f_{11,12} \rightarrow 0$ (as required in the most preferred texture) and the value at $\left|r_{2}\right| \simeq 0.1$ is most important. We find that $U_{e 3}$ is between 0.07 to 0.09 . For this fit, $\sin ^{2} 2 \theta_{23}$ is maximal and $\tan ^{2} \theta_{\text {solar }} \sim 0.4$. The prediction for $\sin \left|\delta_{M N S}\right|$ lies between 0.3 to 0.7 for $\left|r_{2}\right| \simeq 0.1$ as shown in figure 2. The Yukawa matrices are assumed to be hermitian in order to keep the model free from SUSY CP problem.

The presence of $h^{\prime}$ is a necessity to suppress proton decay (suppress $\tilde{A}_{h h}$ ) and fit the fermion masses. This $h^{\prime}$ also helps to explain CKM CP violation [6].

Finally, we show how the above proton decay suppression arises by an adjustment among different VEVs (or symmetry breaking parameters). In the above, we need to have $r_{3} \simeq 0$ in the Eq. (3). Since this is satisfied in Eq.(24) for the SU(5) condition, it may be a hint that we stay close to the $\mathrm{SU}(5)$ symmetric vacuum. Secondly, we need $r_{2} \simeq 0$ while suppressing $\overline{\mathbf{1 2 6}}$ colored Higgs contribution to proton decay, namely only $U_{14}$ is enhanced while other mixings for the sub-multiplets in $\overline{\mathbf{1 2 6}}$ are small,

$$
U_{14} \gg V_{15}, X_{14}, Y_{15}
$$




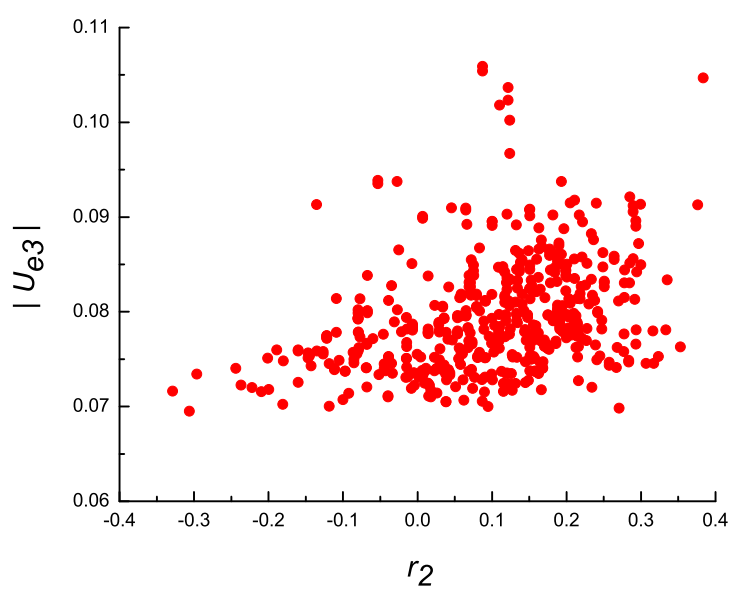

FIG. 1: $\left|U_{e 3}\right|$ is plotted as a function of $r_{2}$.

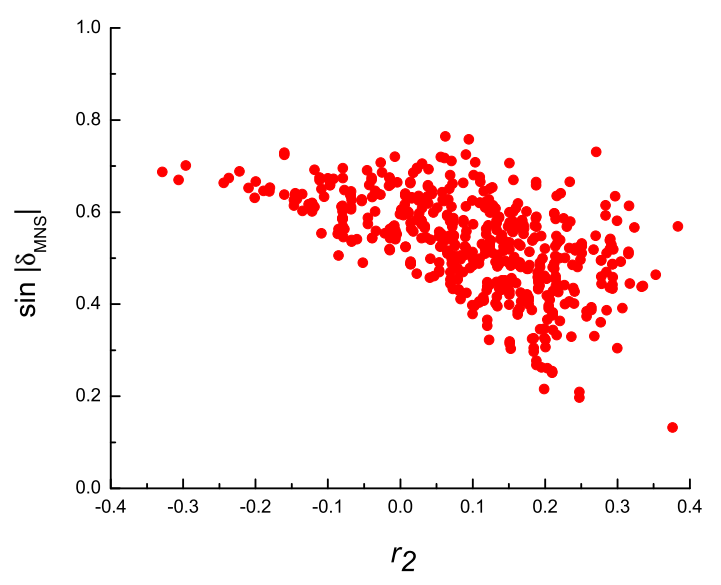

FIG. 2: $\sin \left|\delta_{M N S}\right|$ is plotted as a function of $r_{2}$.

In order to explain how these conditions may be satisfied in the minimal $\mathrm{SO}(10)$ model, we denote the VEVs of the submultiplets in $\mathbf{2 1 0}$ multiplet that break $\mathrm{SO}(10)$ symmetry as follows: $\Phi_{1}:(\mathbf{1}, \mathbf{1}, \mathbf{1}), \Phi_{2}:(\mathbf{1 5}, \mathbf{1}, \mathbf{1})$, $\Phi_{3}:(\mathbf{1 5}, \mathbf{1}, \mathbf{3})$ (where numbers in the parenthesis denote $G_{422}$ quantum numbers). The VEVs $\Phi_{i}$ are around the unification scale of three gauge couplings. Recall that in the $\mathrm{SU}(5)$ symmetric vacua $17,\left[18\right.$, the $\Phi_{i}$ 's satisfy the following relation: $\sqrt{6} \Phi_{1}=\sqrt{2} \Phi_{2}=\Phi_{3}$ (We have used the same normalization as in the Ref.[17]). They lead to the SU(5) relations (20 23). Now we perturb the Higgs potential with a small coupling, $\lambda_{2} H \Delta \Phi$. We obtain $r_{3} \propto \lambda_{1}\left(\sqrt{6} \Phi_{1}-\Phi_{3}\right)$ (where $\lambda_{1}$ is associated with $\lambda_{1} H D \Phi$ term). If $\sqrt{6} \Phi_{1}=\Phi_{3}$, we have $r_{3} \simeq 0$. We obtain the Higgs mixings

$$
\begin{aligned}
& U_{14} \simeq-6 \sqrt{5} \lambda_{2} / \eta \frac{\sqrt{2} \Phi_{2}-\Phi_{3}}{\sqrt{6} \Phi_{1}+\sqrt{2} \Phi_{2}+8 \Phi_{3}}+\cdots, \\
& X_{14} \simeq-2 \sqrt{15} \lambda_{2} / \eta \frac{\sqrt{6} \Phi_{1}-\sqrt{2} \Phi_{2}}{\sqrt{6} \Phi_{1}+3 \sqrt{2} \Phi_{2}+6 \Phi_{3}}+\cdots
\end{aligned}
$$

where $\eta$ is a coupling of $\Phi \Delta \bar{\Delta}$ term. We also have similar terms for $V_{15}, Y_{15}$ and $Y_{16}$. All these terms have different denominators. All the Higgs mixing angles tend to zero in the limit $\lambda_{2} \rightarrow 0$. However, suppose that $\sqrt{6} \Phi_{1}+$ $\sqrt{2} \Phi_{2}+8 \Phi_{3} \sim 0$ is satisfied, only $U_{14}$ can be of finite value, all other mixing angles are zero and Eq.(30) is satisfied. On such a vacuum, the proper strange quark and the muon masses can be realized by an enhancement of $U_{14}$, and Yukawa coupling of up-type quark, $Y_{u}$, is almost proportional to $h$. This is just an example, and in our detailed quantitative work, we keep all other terms in the Higgs potential and we satisfy Eqs. (24130) to suppress the proton decay rate.

\section{CONCLUSION}

In conclusion, we have analyzed the fermion masses and dimension five $\Delta B=1$ operators in the minimal $\mathrm{SO}(10)$ model with $\mathbf{1 0}, \overline{\mathbf{1 2 6}}$ and $\mathbf{1 2 0}$ Higgs fields coupling to matter. We show that by a choice of suitable textures, one can not only get correct fermion masses and mixings but also suppress the contributions to proton decay from both the $L L L L$ and $R R R R$ operators for the entire range allowed $\tan \beta$ parameter of MSSM. This choice of textures requires a suitable $\mathrm{SO}(10)$ breaking vacuum condition which is close to $\mathrm{SU}(5)$ invariant vacua. In the most favorable region of parameter space $U_{e 3}$ is predicted to be 0.07 to 0.09 and $\sin \left|\delta_{M N S}\right|$ to be 0.3 to 0.7 , which can be used to test the model.

\section{Acknowledgments}

This work of B.D. and Y.M. is supported by the Natural Sciences and Engineering Research Council of Canada and the work of R. N. M. is supported by the National Science Foundation Grant No. PHY-0354401.

[1] P. Minkowski, Phys. Lett. B 67, 421 (1977); M. GellMann, P. Rammond and R. Slansky, in Supergravity, eds. D. Freedman et al. (North-Holland, Amsterdam, 1980); T. Yanagida, in proc. KEK workshop, 1979 (unpublished); S. L. Glashow, Cargese lectures, (1979); R. N. Mohapatra and G. Senjanović, Phys. Rev. Lett. 44, 912 (1980).

[2] K. S. Babu and R. N. Mohapatra, Phys. Rev. Lett. 70, 2845 (1993).

[3] D. G. Lee and R. N. Mohapatra, Phys. Rev. D 51, 1353 (1995); L. Lavoura, Phys. Rev. D 48, 5440 (1993); B. Brahmachari and R. N. Mohapatra, Phys. Rev. D 58, 015001 (1998); K. Oda, E. Takasugi, M. Tanaka and M. Yoshimura, Phys. Rev. D 59, 055001 (1999); K. Matsuda, Y. Koide, T. Fukuyama and H. Nishiura, Phys. Rev. D 65, 033008 (2002) [Erratum-ibid. D 65, 079904 
(2002)]; T. Fukuyama and N. Okada, JHEP 0211, 011 (2002); N. Oshimo, Nucl. Phys. B 668, 258 (2003).

[4] B. Bajc, G. Senjanovic and F. Vissani, Phys. Rev. Lett. 90, 051802 (2003).

[5] H. S. Goh, R. N. Mohapatra and S. P. Ng, Phys. Lett. B 570, 215 (2003); Phys. Rev. D 68, 115008 (2003).

[6] B. Dutta, Y. Mimura and R. N. Mohapatra, Phys. Rev. D 69, 115014 (2004).

[7] K. Anderson et al., hep-ex/0402041 M. Apollonio et al., Eur. Phys. J. C 27, 331 (2003); M. V. Diwan et al., Phys. Rev. D 68, 012002 (2003); D. Ayres et al. [Nova Collaboration], hep-ex/0210005 Y. Itow et al. [T2K collaboration], hep-ex/0106019 I. Ambats et al. [NOvA Collaboration], FERMILAB-PROPOSAL-0929 (2004).

[8] B. Dutta, Y. Mimura and R. N. Mohapatra, Phys. Lett. B 603, 35 (2004).

[9] S. Bertolini, M. Frigerio and M. Malinsky, Phys. Rev. D 70, 095002 (2004); W. M. Yang and Z. G. Wang, hep-ph/0406221

[10] For a review, J. Hisano, hep-ph/0004266 B. Bajc, P. Fileviez Perez and G. Senjanovic, Phys. Rev. D 66, 075005 (2002).

[11] T. Goto and T. Nihei, Phys. Rev. D 59, 115009 (1999); H. Murayama and A. Pierce, Phys. Rev. D 65, 055009 (2002).

[12] N. Sakai and T. Yanagida, Nucl. Phys. B 197, 533 (1982); S. Weinberg, Phys. Rev. D 26, 287 (1982).

[13] K. S. Babu and S. M. Barr, Phys. Rev. D 65, 095009 (2002); K. S. Babu, J. C. Pati and F. Wilczek, Nucl.
Phys. B 566, 33 (2000); R. Dermisek, A. Mafi and S. Raby, Phys. Rev. D 63, 035001 (2001).

[14] H. S. Goh, R. N. Mohapatra, S. Nasri and S. P. Ng, Phys. Lett. B 587, 105 (2004).

[15] T. Fukuyama, A. Ilakovac, T. Kikuchi, S. Meljanac and N. Okada, JHEP 0409, 052 (2004).

[16] T. E. Clark, T. K. Kuo and N. Nakagawa, Phys. Lett. B 115, 26 (1982); C. S. Aulakh and R. N. Mohapatra, Phys. Rev. D 28, 217 (1983).

[17] T. Fukuyama, A. Ilakovac, T. Kikuchi, S. Meljanac and N. Okada, hep-ph/0405300

[18] C. S. Aulakh, B. Bajc, A. Melfo, G. Senjanovic and F. Vissani, Phys. Lett. B 588, 196 (2004); B. Bajc, A. Melfo, G. Senjanovic and F. Vissani, Phys. Rev. D 70, 035007 (2004).

[19] C. S. Aulakh and A. Girdhar, hep-ph/0204097

[20] G. Lazarides, Q. Shafi and C. Wetterich, Nucl. Phys. B 181, 287 (1981); R. N. Mohapatra and G. Senjanovic, Phys. Rev. D 23, 165 (1981).

[21] S. M. Barr, Phys. Lett. B 112, 219 (1982); J. R. Ellis, J. S. Hagelin, S. Kelley and D. V. Nanopoulos, Nucl. Phys. B 311, 1 (1988).

[22] B. Dutta, Y. Mimura and R. N. Mohapatra, in preparation.

[23] R. Dermisek et al. in [13]; K. Turzynski, JHEP 0210, 044 (2002); D. Emmanuel-Costa and S. Wiesenfeldt, Nucl. Phys. B 661, 62 (2003). 